\title{
Bioética de las innovaciones genéticas y la inteligencia artificial
}

\author{
Never let me go | Mark Romanek | 2010 \\ Ex machina | Alex Garland | 2015 \\ Celeste Bogetti* \\ Universidad Nacional de Mar del Plata
}

Recibido: 26 de septiembre 2016; aprobado: 10 de noviembre 2016

\begin{abstract}
Resumen
El presente trabajo tiene como objetivo abordar dos filmes: Never let me go (2010) y Ex-Machina (2015). Se propone para la lectura de los mismos el establecimiento de un contrapunto entre ambos, teniendo como eje la consideración de la dignidad y del estatuto de humanidad al que este concepto viene adherido. En este marco el cine aparece como herramienta para plantearnos dilemas éticos y bioéticos. La aplicación de nuevas tecnologías en seres humanos da lugar a nuevos dilemas en el ámbito biomédico, lo cual plantea dilemas bioéticos. Dentro de dichas tecnologías se encuentran la manipulación genética/clonación y la generación de inteligencia artificial, temas abordados por las mencionadas películas. Con respecto a estas pueden plantearse distintos interrogantes: ¿Tienen los seres humanos, creados artificialmente para un fin determinado, la misma dignidad que otros? ¿Cómo se argumenta moralmente la intervención de la tecnología para dar lugar a humanos que sean medios para un fin? ¿Cuál es el objetivo de la inteligencia artificial? ¿Puede reemplazar está a los seres humanos? ¿Puede atribuírseles la cualidad de la dignidad? Puede pensarse a estos dilemas éticos, que giran en torno a la humanidad y a la dignidad, interrogadas por las intromisiones de las nuevas tecnologías, como una irrupción de singularidad en un universo donde el hombre era considerado finito y perecedero, ¿y si aparece la posibilidad de la inmortalidad?
\end{abstract}

Palabras clave: Bioética | Cine | Biotecnología | Inteligencia artificial | dignidad | humanidad

Bioethics of genetic innovations and artificial intelligence

\begin{abstract}
This article addresses two films: Never let me go (2010) and Ex-Machina (2015). It proposes an analysis of these, establishing a counterpoint between both of them, having as axis for this analysis the dignity and the condition of humanity to which that concept is linked. In this context, cinema appears as a tool for proposing ethical and bioethical dilemmas. The aplication of new technologies in human beings brings new dilemmas in the biomedical area, which raises bioethical dilemmas. Within these technologies we find genetic manipulation/cloning and artificial intelligence, topics that both movies approach. Regarding this, different questions can be posed: do the human beings, created artificially for an especific purpose, have the same dignity as others? How the intervention of technology to create humans that are means to an end can be morally justified? Which is the objective of creating artificial intelligence? Could it replace human beings? Could it be considered as having dignity? We can think about this ethical dilemmas, that revolve around humanity and dignity, questioned by the intromission of new technologies, as an irruption of singularity in an universe where the mankind is considered mortal and finite, what if the posibilty of inmortality arises?
\end{abstract}

Keywords: Bioethics | Cinema | Biotechnology | Artificial intelligence | Dignity | Humanity

Artificio y naturaleza no son ya categorías opuestas (claro, nunca lo han sido, pero jamás su proximidad habia sido tal: ambos se confunden) (Le Breton, 2007), entonces (...) ¿Qué es lo que define al ser bumano como tal? (Cambra Badii, 2013, p. 37)

\section{Introducción}

El presente trabajo tiene como objetivo abordar dos filmes: Never let me go (2010) y Ex-Machina (2015). Los mismos ya han sido descriptos y analizados individualmente por otros autores (el primero por Cambra Badii,
2010, 2012 y Gómez García, 2011; y el segundo por Goldman y Ramallo, 2015 y García Manrique, 2016), por lo cual la novedad que se propone para la lectura de los mismos es el establecimiento de un contrapunto entre ambos, teniendo como eje la consideración de la dignidad y del estatuto de humanidad al que este concepto viene adherido.

La aplicación de nuevas tecnologías en seres humanos da lugar a nuevos dilemas en el ámbito biomédico, lo cual plantea dilemas bioéticos. Dentro de dichas tecno-

*celes.bogetti@gmail.com 
logías se encuentran la manipulación genética y la clonación, que puede tener diversos fines, y la generación de inteligencia artificial, que así mismo puede ser aplicada para lograr distintos objetivos.

En la película Never let me go se puede observar la manipulación genética/clonación con el propósito de dar lugar a sujetos que sirvan como donantes de órganos. En Ex-Machina se relata la creación de una inteligencia artificial (IA) con fines pocos claros en cuanto a su aplicación concreta, pero que involucran a otros sujetos para su testeo, y que dejan vislumbrar efectos sobre el futuro de los seres humanos.

En ambas puede pensarse el lugar del concepto de la dignidad humana mediante distintos interrogantes: $¿$ Tienen los seres humanos, creados artificialmente para un fin determinado, la misma dignidad que otros? ¿Cómo se argumenta moralmente la intervención de la tecnología para dar lugar a humanos que sean medios para un fin? ¿Qué explicaciones psicológicas estarían operando para esto? ¿Cuál es el objetivo de la IA? ¿Puede reemplazar está a los seres humanos? ¿Si se logra una IA con equivalente capacidad a la de un ser humano -como se plantea en la película Ex-machina, en caso de que la IA creada pase el test de Turing- podemos comenzar a debatir sobre el estatuto de su dignidad?

El cine aparece entonces como un instrumento para poder pensar y plantearnos estos dilemas bioéticos, promoviendo el pensamiento ético (Fariña y Solbakk, 2012) interpelándonos como espectadores a través de la matriz ético-estética que propone (Gómez, Michel Fariña y Solbakk, 2011).

\section{La dignidad y la humanidad cuestionadas}

Ambos filmes presentan interrogantes en torno al estatuto de humanidad de los sujetos que son protagonistas de las historias. Estos interrogantes vienen de la mano de los avances tecnológicos y el impacto de estos en los cuerpos de los sujetos, que generan justamente esta necesidad de poner entre paréntesis o problematizar la condición humana.

Como plantea Cambra Badii (2013) "el cine es un vehículo fundamental del imaginario tecnológico y permite representar adelantos existentes o posibles teniendo en cuenta la realidad tecnológica circundante y las posibilidades que brindan las tecnologías de la imagen y el sonido” (p. 35-36). A través del cine, entonces, podemos representarnos estos posibles futuros, más o menos ve- rosímiles, e interrogarnos al respecto, planteando la pregunta desde la estructura de un dilema.

El dilema presenta la propiedad de "enfrentarnos con opciones previstas por los cursos de acción disponibles. El método ético radica en no precipitar una opinión respecto de alguna de ellas, sino transformar el dilema en un problema, es decir en una empresa de pensamiento" (p. 17, Michel Fariña, 2012).

Se puede partir entonces, de tener en cuenta desde qué nociones dignidad y humanidad nos posicionamos, para luego poder establecer como problematizar en torno a los dilemas que estos filmes nos proponen.

En cuanto a la dignidad, desde un plano legal, puede apelarse a la conceptualización propuesta en la Declaración Universal de Derechos Humanos (1948) en la cual se generaliza esta cualidad a la totalidad de los seres humanos sin distinción de origen, raza, condición social y económica, orientación sexual, etc.

Desde el plano filosófico puede tomarse la definición kantiana del concepto, según la cual se establece que "en el reino de los fines todo tiene o un precio o una dignidad. Aquello que tiene precio puede ser sustituido por algo equivalente, en cambio, lo que se halla por encima de todo precio y, por tanto, no admite nada equivalente, eso tiene una dignidad. (...) aquello que constituye la condición para que algo sea fin en sí mismo, eso no tiene meramente valor relativo o precio, sino un valor interno, esto es, dignidad.” (Kant, 1785, p. 47-48).

En síntesis, será entendida como una cualidad intrínseca y atribuida a todos los seres humanos, que se define por la imposibilidad de reemplazar a un sujeto por otro, y por poseer este un valor tal que impide semejante transacción.

La noción de humanidad conlleva otras dificultades. Chavarría Alfaro (2015) plantea que "al usar el término identidad humana se hace referencia a la noción genérica de hombre (en el sentido que engloba lo masculino y lo femenino)" (p. 97). Esta identidad incluye compartir la herencia de la especie, la misma unidad cerebral, la aptitud para hablar. Con la irrupción de las nuevas tecnologías, específicamente la cibernética y la biotecnología, se producirá una intervención sobre los humanos que permitirá replantearse su condición de tales.

Por esto se interroga Cambra Badii (2013) al preguntarse “¿Qué consideramos qué es el ser humano?” (p. 37), respondiendo a esto con la alusión al concepto de poshumano.

El poshumanismo está dado por la relación del humano con las posibilidades tecnológicas y las conse- 
cuencias que esto conlleva. "Una de las consecuencias más relevantes del desarrollo de la cibernética es la descorporeización de la razón, pues el razonamiento ya no era exclusivo del cuerpo humano, sino que podía ser reproducido y copiado fuera del cuerpo por cualquier máquina inteligente” (p. 99, Chavarría Alfaro, 2015). En cuanto a la biotecnología "es el fruto de los progresos contemporáneos de la biología molecular y la genética” (p. 101, Chavarría Alfaro, 2015). Esta refiere a dos cuestiones concretas, las relacionadas con conocimiento y manipulación en células de origen animal y vegetal, por un lado, y con células de origen humano (manipulación genética y técnicas de reproducción) (Chavarría Alfaro, 2015).

En función de estos comentarios teóricos puede comenzarse a indagar en torno a la posibilidad de aplicar la noción de dignidad a seres producto de la cibernética o de la biotecnología, que son seres pero, según estas distinciones, clasificables dentro del grupo de los "poshumanos". ¿Tienen estos poshumanos el derecho a recibir el mismo estatuto de dignidad? Si la dignidad pareciera ser la potestad que logró adquirir el ser humano de la modernidad, ¿puede compartirse esta característica con los seres posthumanos de la postmodernidad?

Desde otra perspectiva, para interrogarnos por la condición de humanidad, Kletnicki (2006) hace alusión al plano simbólico y al núcleo real en cuanto a la definición de dicho concepto. El primero refiere a lo que delimita la subjetividad de una época, el segundo a lo que es necesario para salvaguardar las características estructurales de la especie. La humanidad aparece ligada a ciertos términos como "el origen de la vida, la sexualidad, la reproducción, la transmisión de la herencia biológica y cultural, la lógica de los lazos de parentesco (...)” (p. 431). También podría agregarse la finitud dentro de esta lista.

La mayoría de estas cuestiones parecen estar ausentes en las historias de los personajes que narran los filmes propuestos. En Never let me go la cuestión de la finitud de los donantes (cuya condición de humanos es cuestionada por el tratamiento que se les $\mathrm{da}$ ) aparece como un mero medio para evitar la finitud del resto de la población, considerados humanos de pleno de derecho. En Ex-Machina, la inmortalidad se plasma en la figura de la IA, Ava, que es un ser construido y no procreado. Ava conoce su origen, pero no su destino, sabe que puede ser inmortal -porque siempre puede repararsepero avizora su destrucción por las intenciones de su creador. Los donantes no son construidos, sino creados artificialmente por procesos de procreación artificial, pero desconocen su origen concreto -de dónde provienen, cuestión que resuelven parcialmente con la idea de los 'posibles', aquellos humanos de los que serían copias-. Podemos ver como entonces en ambos filmes faltarían cuestiones propias de este núcleo real, de este conglomerado de rasgos que hacen que podamos definir como humanos a estos seres. Parafraseando el planteo de Kletinicki (2006) ¿Hasta qué punto podemos considerar esos rasgos que comienzan a faltar como cambios en el plano de lo simbólico, y por ende en el estatuto de humanidad, y hasta donde considerar que los mismos afectan particularidades del núcleo real irreductibles para pensar en un ser como humano?

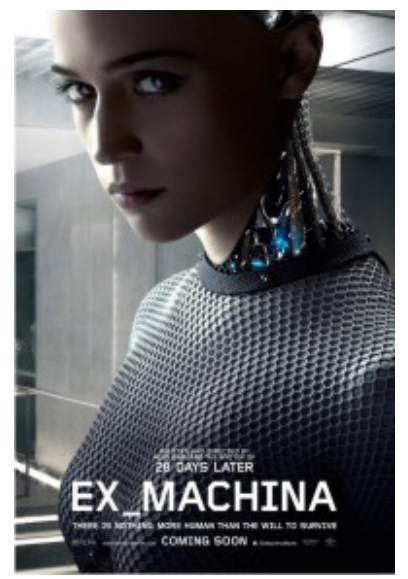

Se pueden entonces plantear interrogantes sobre lo siguiente: en Never let me go sobre los seres humanos creados a partir de intervenciones de la biotecnología, específicamente el uso de clonación y manipulación genética en el marco de técnicas de reproducción asistida (TRA). Existe en la actualidad acuerdo en considerar que los seres humanos engendrados a través de la implementación de TRA son poseedores de igual grado de humanidad que cualquier otro ser humano producto de una concepción no intervenida tecnológicamente. Por ende son así mismo portadores de dignidad. Y si bien la manipulación genética y la clonación no son procedimientos con respecto a los cuales exista consenso, y que más bien generan cierto grado de rechazo, si seres humanos surgieran de dichos procedimientos parece improbable que se les negara el reconocimiento de su condición de tales.

A pesar de esto, Never let me go nos sitúa en un escenario donde los donantes, a los cuales reconocemos claramente como seres humanos, no son considerados iguales que los demás personas. Estos son producto de la implementación de tecnologías de reproducción, sin que nos quede clara si los mismos han sido fruto de 
manipulación genética y/o la clonación, si ha habido una gestante de esos seres, o han sido desarrollados también en un tipo de ambiente no humano (al estilo de las bolsas en que eran gestados los personajes de la película La Isla, que funcionaban a modo de una matriz externa).

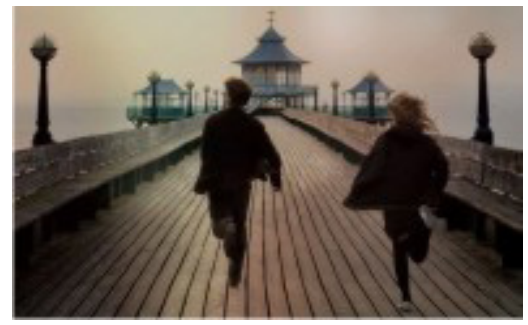

Tomando la definición de dignidad kantiana, no son un fin en sí mismos ni tienen un valor intrínseco, son intercambiables, tienen un propósito concreto donar órganos- y seguramente, aunque no se establece explícitamente, tienen un precio.

En cuanto a Ex-machina se verán dos posturas contrapuestas, la de Nathan, el creador de Ava, quien la considera reemplazable y mejorable, motivo por el cual planea destruirla para crear una versión mejor (como ya ha creado prototipos anteriores); y la de Caleb, el empleado de la empresa de Nathan que es convocado para testear, a través de la prueba de Turing, a esta IA.

Caleb comienza viendo a Ava como una máquina, $\mathrm{y}$ leyendo en sus acciones y sus interlocuciones un procesamiento maquínico, para ir a lo largo del filme cambiando su perspectiva y atribuyendo a Ava una entidad tal, una humanidad tal podría decirse, que lo lleva a querer salvarla del desmantelamiento que Nathan ha programado para ella.

Pueden recortarse, a través de la lectura ético-clínica de los filmes, los dilemas ante los que estos protagonistas se ven enfrentados, y mediante este abordaje buscar salir de una situación dicotómica de a favor o en contra de una $\mathrm{u}$ otra de estas posiciones, para poder seguir pensando (Michel Fariña y Solbakk, 2012).

\section{Lectura ético-clínica de dilemas bioéticos en los filmes Never let me go y Ex-machina}

\section{Never let me go}

Como se observa a lo largo de la película, los donantes son humanos, al menos física y fisiológicamente. Puede ponerse en discusión su constitución subjetiva como tales, ya que sorprende a lo largo del filme la actitud pasiva de estos seres ante su destino. Surge el interrogante sobre cómo no ven otra salida que la de participar en el marco de esas donaciones, que no pueden entenderse como tales ya que les falta algo fundamental, el componente voluntario y altruista. Esto conlleva una pregunta por otro tema, que es la autonomía de los sujetos. Puede plantearse hasta qué punto, si bien no se resisten a participar de las donaciones, tienen capacidad de auto determinarse, en base a los fundamentos establecidos por su formación durante su crecimiento, y la consideración $\mathrm{y}$ tratamiento que reciben de su entorno.

Con respecto a esto puede pensarse en la crianza y los efectos de la misma en los psiquismos de estos sujetos, que hace que se encuentren tan apacibles y entregados a su inexorable destino. Los donantes son moldeados a través del lenguaje y la programación de sus mentes de modo tal que no realicen ningún tipo de acción contra el orden establecido, los protagonistas "han perdido la capacidad de rebelarse contra el sistema" (p. 58, Gómez García, 2011).

Este rasgo de humanidad que se demanda a estos seres, que es la acción de confrontar, escapar o rebelarse al inmodificable fin de ser donantes, no aparece en toda la historia hasta el momento en la cual la misma da un giro. Finalmente dos de sus protagonistas logran unirse amorosamente. Luego de haber esperado años, consiguen concretar su encuentro, pero una cuestión se interpone en lo que sería un final feliz -dentro del marco de posibilidades de estos sujetos-, dado que uno de ellos, Tommy, ya se encuentra cumpliendo su función de donante. Allí entra en escena la figura del 'aplazamiento', esta posibilidad de diferir las donaciones para poder así, compartir un período mayor de tiempo juntos. El criterio para solicitar esto es poder probar que existe un amor verdadero entre los miembros de la pareja. Los personajes, Kathy y Tommy, buscan la salida dentro de las reglas de ese mismo sistema que los colocó en ese lugar entre la vida y la muerte, en ese lugar de objetos para servir a la prolongación de la vida de otros.

Se ve entonces que este universo en que ellos viven se encuentra conmovido por la existencia de algo que los mueve a alejarse de su incuestionable finalidad. Se observa una situación que puede encuadrarse en un esquema de A (afirmación) y -A (negación). Puede ubicarse en el primer grupo (A) la aceptación con resignación del destino que les toca, que es algo invariable. En el segundo $(-\mathrm{A})$, puede plantearse la posibilidad de rebelarse, de escapar, de resistirse (que si bien nunca se explicita, se desliza en esos viajes a la playa, cerca del barco encallado, 
que dan cuenta de la existencia de la posibilidad de la huida, sea a través del escape o del suicidio).

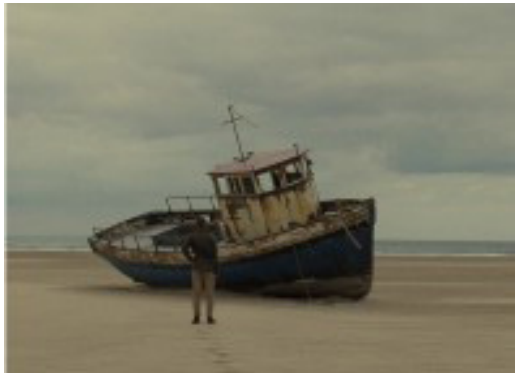

Este es el universo de Tommy y Kathy, y es dentro de los parámetros de este en el que se mueven para encontrar una opción, la de pedir el aplazamiento. No se salen de las normas de su sociedad, no se conforman pero no se rebelan. Es decir que siguen funcionando en esta misma lógica. Es por esto, que al recibir una negativa a su pedido de postergación de las donaciones, siguen ateniéndose a las pautas del sistema y retornan al hospital, como si hubieran agotado todos los recursos. Puede decirse que en esta instancia los personajes se mueven dentro la categoría de lo universal.

Es aquí donde se produce algo que queda por fuera de este sistema, que rompe con esta aceptación acrítica de las circunstancias, con esta calificación que se hace de ellos como entes acerca de los cuales se debe comprobar si tienen o no alma, a estos no humanos, iguales pero diferentes de los demás habitantes de esa sociedad.

En el viaje de regreso al hospital Tommy pide bajarse del auto en que se dirigían a dicho lugar, y, como lo hacía cuando niño (conducta que le es cuestionada y la cual él termina suprimiendo en el marco de su educación), grita y llora (esto se denomina "tantrum" en inglés, idioma original del libro y su homónima adaptación cinematográfica, que significa que tiene un "ataque de cólera, de rabia"). Puede verse emerger entonces algo nuevo, diferente, otra salida a la situación, una singularidad. Sigue estando presente la inexorabilidad del destino, pero se esfuma la pasividad (al menos por un momento), aparece el sujeto que se queja, que siente angustia, que no se resigna y no acepta lo que va a pasarle. Por eso llora y grita, porque no quiere experimentar ese desenlace. Aparece allí Tommy como sujeto, como humano, no solo corporalmente sino psicológicamente. Su singularidad retorna en la expresión de una conducta, una queja subjetiva, por un presente que lo daña y por un futuro que ve coartado, a través de algo -el ataque de rabia-que todo ese procesamiento y manipulación mental no pudieron borrar, aunque así hubiera parecido.

Resulta interesante resaltar como esta singularidad emerge no frente a la confrontación del sujeto con la posibilidad de su muerte (no entra en la dicotomía entre morir sin luchar o pelear para conservar su vida), sino que aparece ante la pérdida de la oportunidad de vivenciar por algún tiempo más, una relación amorosa con la persona de la que ha estado enamorado toda su vida.

\section{Ex-machina}

En Ex-machina somos espectadores de la historia de tres personajes principales, Nathan, Caleb y Ava. En un primer momento vemos a Caleb arribar a la vivienda de su excéntrico jefe, Nathan, y enterarse que ha sido convocado allí por motivos diferentes de los que él creía. Su función será aplicar el test de Turing a una IA creada por su jefe.

En esta etapa de la historia Caleb conoce a Ava, la IA, y está convencido del estatuto de máquina de esta, y de su diferencia con ella, él es humano.

$\mathrm{Al}$ avanzar la trama, se irán mostrando los sucesivos encuentros entre Caleb y Ava, supervisados siempre por Nathan. Durante los mismos Caleb empezará a reconocer que desarrolla sentimientos por Ava, a dudar sobre si ella tiene sentimientos -cuestión de la que, al inicio, estaba seguro que no era así-. Esta confusión entre humano y máquina escalará hasta el punto de generarle dudas sobre su condición, es decir, sobre si él sigue siendo humano o es una máquina. Necesitará para corroborar esto lastimarse el brazo con un objeto filoso y verse sangrar.

Hasta aquí se ha visto a un Caleb que se caracteriza por creer que Ava es una máquina (realmente sorprendente y mucho más sofisticada y compleja de lo que él imaginaba posible) y por cumplir con su trabajo, de aplicar la prueba. También aparece maravillado por la vida de su jefe, y la expectativa de haber sido convocado por alguien a quien admira profesionalmente.

Un acontecimiento cambiará los eventos y las prioridades de Caleb: la amenaza sobre la 'vida' de Ava. Esto genera en el personaje un gran debate interno, ya que debe contraponerse a su jefe, perder seguramente su trabajo y quedarse sin posibilidad alguna de llegar a volver a desempeñarse laboralmente en ese nivel. A esas alturas se encuentra muy involucrado con Ava, se ha comenzado a convencer realmente de que ella tiene conciencia, sentimientos, y sobre todo de que tiene miedo de 'morir'. 
Finalmente, él decide actuar acorde a lo que cree que es lo correcto, también en parte acorde a sus sentimientos personales. Él sabe que Ava es una máquina pero a partir de su interacción con ella, para él 'tiene vida', porque tiene una mente a través de la que expresa sus pensamientos, da cuenta de su personalidad, y también expresa sentimientos (por ejemplo lo quiere a él y le teme a Nathan). Esto le da estado de ser individual e irremplazable desde su mirada, ¿podría pensarse que también es pasible de que se le atribuya la categoría de dignidad? Esto conlleva que Caleb accione de un modo impensado para él cuándo llegó a la casa/laboratorio de Nathan. Se enfrenta a su jefe y busca salvar a Ava.

Caleb deja de accionar dentro de lo universal, que "consiste en que, a determinado tipo de hombre, corresponde decir u obrar determinada clase de cosas según lo verosímil o lo necesario" (p. 32, Solbakk, 2012). Se da un momento de singularidad que rompe este universal, que es actuar hacia una máquina como hubiera actuado si peligrara la integridad de otro ser humano. Esto permite inferir el valor que da Caleb a la existencia de Ava. Caleb va claramente mucho más allá de lo que sabe, como experto en el tema de la tecnología y la programación informática, para dar respuesta ante esa situación que pone entre 'la vida y la muerte' a la máquina a la que estaba evaluando.

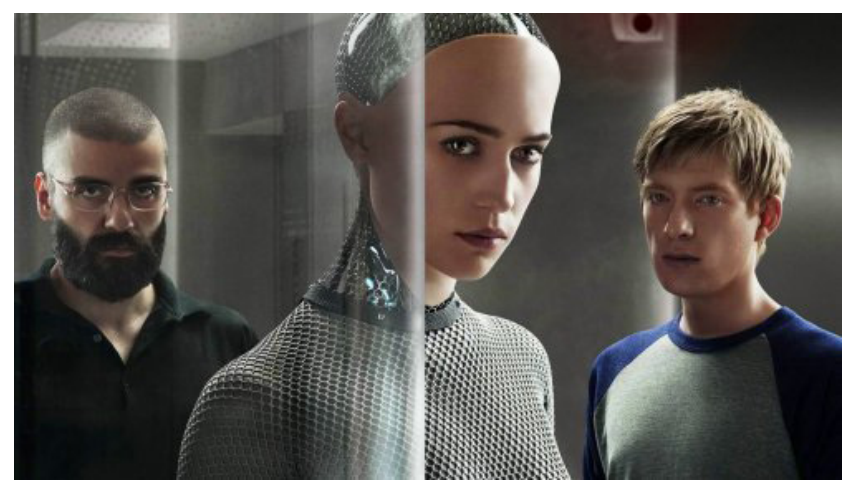

Para concluir, puede decirse decir que Caleb se encuentra en el plano de la 'decisión', no de la opción ni la elección. Esto supone que él se confronta con algo sobre lo cual no tiene garantías, ya que no hay conocimiento previo en que apoyarse -nunca para él una maquina ha sido más que tal cosa, sabe de máquinas pero no de aquellas que puedan confundirlo con respecto a sus condiciones humanas-. Al no existir antecedentes esto no le permite tampoco calcular previamente lo que va a hacer. Se ve con claridad en la película como él va armando su decisión 'sobre la marcha'. "No hay allí un saber que pueda operar como referencia más cerrada o más amplia, sino la necesidad de una intervención subjetiva que produzca una novedad en la estructura de la situación” (p. 99, Kletnicki, 2010).

\section{Comentarios finales}

En ambos relatos encontramos ciertos componentes comunes, como la posibilidad de plantearnos el enfrentarnos ante la muerte a través de distintos avances tecnológicos, los de las biociencias y los de la cibernética. Así mismo, son estos avances y esta posibilidad de enfrentarnos e inclusive vencer a la muerte (Goldman y Ramallo, 2015), lo que trae otra cuestión que comparten estos filmes, la pregunta por la condición humana y la dignidad de esta humanidad, o de esta nueva humanidad.

Las intervenciones biotecnológicas sobre el cuerpo y la mente humana muestran la posibilidad de utilizar la clonación/manipulación genética para generar seres más perdurables, o como se relata en Never let me go, generar donantes interminables para reemplazar los órganos todas las veces que sea necesario y así prolongar las vida indeterminadamente. La cibernética nos plantea otra posibilidad, la de poder aislar la conciencia en un ser físicamente imperecedero, Ex-machina nos presenta otra faceta, la creación de un ser completamente artificial pero que puede pasar por humano o ¿volverse humano a través del aprendizaje?

La cuestión de la dignidad atraviesa ambas propuestas, en la primera vemos a seres claramente humanos, a pesar de la modalidad de su concepción, ser considerados meros objetos, carentes de dignidad. En la segunda vemos una máquina, claramente no humana, ser atribui$\mathrm{da}$ con sentimientos, pensamientos propios y derecho a vivir, es decir, reconocida en su dignidad como ser singular y único, no siendo aceptable su destrucción por ningún motivo.

Ambas películas se plantean en el plano de lo distópico, nos muestran posibilidades que si bien no son reales, son al menos verosímiles, pero indeseables. ¿Llegaremos a considerar a las personas creadas mediante la aplicación de ciertas biotecnologías y técnicas de reproducción clonación, manipulación genética- como menos humanas que aquellas que provienen de concepciones naturales? ¿Será eso coincidente con la capacidad de mostrar empatía y atribuir estatuto humano a una máquina, por más similar que sea su comportamiento al de un humano?

Kletnicki (2006) hace un planteo en cuanto a la intervención tecnológica con respecto a la procreación 
de seres humanos, específicamente mediante el uso de técnicas de reproducción asistida, y la afectación sobre la condición humana que estas producen. Este planteo puede extrapolarse y extenderse al impacto de las tecnologías en la creación o imitación (el caso de las IA) de lo humano. El autor se interroga "¿en qué situaciones esos desarrollos constituyen valiosas mediaciones instrumentales, y en cuáles dichas transformaciones nos confrontan con un límite en el que se pone en cuestión la condición misma de la especie humana?” (p. 430).

El uso de las nuevas tecnologías interpela la humanidad, porque la interviene, la modifica, al menos como la conocíamos hasta ahora. ¿Es la búsqueda del hombre por vencer la muerte lo que lo aleja de lo humano (Goldman y Ramallo, 2015)? ¿Son estos humanos intervenidos por la ciencia humanos? ¿Son los poshumanos menos humanos?

A todas estas preguntas podemos respondernos con lo que plantean Goldman y Ramallo (2015): "tal vez sea más preciso pensar que estamos frente a un nuevo hombre, o un ex-hombre (...)". Esta noción de 'ex' refiere a algo que está más allá de o fuera de. Este hombre del que hablamos, es un hombre que está más allá de sí mismo o inclusive fuera de sí mismo, y al salir de su universal, al aparecer como singularidad es que se ve cuestionada su esencia y reconocimientos más básicos, como la dignidad.

Otro aporte en este tema es el de Baudrillard (2002), quien plantea la inmortalidad como una fantasía, la cual encuentra un intento de realización a través de la ciencia y la tecnología. Este autor, como otros citados, propone las características de muerte y sexualidad como propias de los humanos (entre otros seres vivos), y también como rasgos de evolución de esta especie. La posibilidad de la inmortalidad, a través de las intervenciones tecnológicas, resulta entonces una involución de los seres humanos, ya que los despoja de estos atributos que los hacen lo que son. Es en este sentido que explicita "como ya hemos disociado la reproducción del sexo, intentamos disociar la vida de la muerte" (p. 10, Baudrilliard, 2002).

A través de la manipulación genética, la clonación y el desarrollo de IA dejamos de lado sexualidad como intervieniente en la procreación de los seres, dándole este lugar a la tecnología, y podemos postergar asimismo la muerte de estos, ya que están diseñados para ser imperecederos. Esto nos permite nuevamente interrogarnos, remitiéndonos al planteo de Kletnicki (2006), sobre si estas creaciones pueden seguir siendo definidas como huma- nos, o hablaríamos ya de 'ciberhumanos' (parafraseando la idea de Baudrilliard de la posibilidad de "cibermuerte" que podrían atravesar estos seres, que es un tipo de finitud simulada y a la que se puede acceder para experimentar algo que ya no forma más parte de los cánones que definen a los humanos).

A modo de cierre puede formularse un última reflexión sobre el tema desde la propuesta de Lewkowicz, quien plantea que "una singularidad tiene dos dimensiones, una positiva y una negativa: la negativa es el punto en que el universo lo abandona, no da lugar, no lo reconoce como tal. La positiva se da existencia sin tomar recurso de ese universo" (material interno del curso Cine y Subjetividad, teórico de Lewkowicz compilado por Gil Miranda, 2004).

El aspecto negativo de la singularidad se encuentra en la posibilidad de inmortalidad, la cual no cabe dentro de las categorías que definen la humanidad, del núcleo real que la estructura. El aspecto positivo se observa en este abandono de la finitud, sin tomar como referencia a este universo de seres mortales. La cuestión está en pensar que más se pierde cuando se sale del universo, que más se deja cuando se abandona la finitud, ¿̇se pierden también la humanidad y la dignidad?

Pueden pensarse estos dilemas bioéticos, que giran en torno a la humanidad y a la dignidad siendo interrogadas por las intromisiones de las nuevas tecnologías, como una irrupción de singularidad en un universo donde el hombre era considerado finito y perecedero, ¿y si aparece la posibilidad de la inmortalidad? "al universo se le declara una singularidad y deja de ser universo" (Lewkowicz compilado por Gil Miranda, 2004).

La aparición de esta singularidad, que es la posibilidad de inmortalidad, o mejor dicho la fantasía o deseo tecnológico de inmortalidad del que habla Baudrilliard (2002), aparece como una ruptura, o por el momento un intersticio en ese universo -que avizora una disolución del mismo- que conllevará el cese de las reglas y características del núcleo real de la humanidad, es decir, la culminación de la existencia de este universo, de este humano, tal como lo conocíamos. Con el surgimiento de esta singularidad, de este nuevo humano; $u$ otros modos en que se lo ha referenciado, como 'poshumano', 'ciberhumano', 'ex-humano' (el aspecto de su denominación será otro de los temas a definir en su caracterización); vendrá la necesidad de re-definir o inventar las categorías que darán cuentan del núcleo real de esta creación o innovación en la vida, que ya no es más la vida humana dentro del universo de la mortalidad. 


\section{Referencias}

Baudrilliard, J. (2002). La solución final: la clonación más allá de lo humano e inhumano. En La Ilusión vital. Buenos Aires: Siglo XXI.

Cambra Badii, I. (2011). Angustia de clonación. Journal de Ética y Cine, 1(1), 49-58.

Cambra Badii, I. (2012). La angustia ante la finitud. Transplante de órganos y clonación humana. En (Bio)ética y cine: tragedia griega y acontecimiento del cuerpo, Michel Fariña, J. J. \& Solbakk, J. H [Comps.]. Buenos Aires: Letra Viva.

Cambra Badii, I. (2013). La condición poshumana: camino a la integración hombre-máquina en el cine y en la ciencia. Journal de Ética y Cine, 3(1), 35-39.

Chavarría Alfaro, G. (2015). El posthumanismo y los cambios en la identidad humana. Reflexiones, 94(1), 97-107.

García Manrique, R. (2016). Ex machina, o sobre la dimensión corporal de lo humano. Revista de Bioética y Derecho, 37, $171 \rrbracket 176$.

Gil Miranda, S. [Comp.] (2004). Teórico de la asignatura Psicología, Ética y Derechos Humanos, dictado por I. Lewkowicz. Material interno del Curso Cine y Subjetividad, 2016. Recuperado de http://www.elsigma.com/literatura/r...

Goldman, D. \& Ramallo, G. (2015). Desarrollos psicoanaliticos sobre sexualidad y muerte en base a la inteligencia artificial: Pasaje del hombre al ex hombre. Congreso On Line 2015 de Ética y Cine. Recuperado de http://www.eticaycine.org/Ex-machin...

Gómez, M., Michel Fariña, J. J. \& Solbakk, J. H. (2011). Ética y Cine: un moderno teatro griego. Journal de Ética y Cine, 1(1), 9-12. Gómez García, I. (2011). Nunca me abandones. El precio de las donaciones. Revista de Bioética y Derecho, 23, 56-63.

Kant, I. (1785). Fundamentación de la metafísica de las costumbres. San Juan de Puerto Rico: Edición de Pedro M. Rosario Barbosa [1ra Ed. 1921].

Kletnicki Armando (2006). Tecnologías de reproducción asistida: ética y desarrollo científico. XIII Jornadas de Investigación y Segundo Encuentro de Investigadores en Psicología del Mercosur. Buenos Aires, agosto 2006.

Kletnicki, Armando (2010). Ética y decisión. II Congreso Internacional de Investigación y Práctica Profesional en Psicología XVII Jornadas de Investigación Sexto Encuentro de Investigadores en Psicología del MERCOSUR. Buenos Aires, noviembre 2010.

Michel Fariña, J. J. \& Solbakk, J. H. (2012). (Bio)ética: el cine como moderno teatro griego. En (Bio)ética y cine: tragedia griega y acontecimiento del cuerpo, Michel Fariña, J. J. \& Solbakk, J. H [Comps.]. Buenos Aires: Letra Viva.

Organización de las Naciones Unidas. (1948). Declaración Universal de los Derechos Humanos.

Solbakk, J. H. (2012). Ética y responsabilidad: el pensamiento de la Grecia Clásica y sus lecciones sobre bioética contemporánea. En (Bio)ética y cine: tragedia griega y acontecimiento del cuerpo, Michel Fariña, J. J. \& Solbakk, J. H [Comps.]. Buenos Aires: Letra Viva. 